\title{
Forced and Unforced Unsteadiness in an Axial Turbomachine
}

\author{
Fernández Oro, J.M.; Blanco Marigorta, E.; \\ Argüelles Díaz, K.M.; Ballesteros-Tajadura, R.
}

Universidad de Oviedo, Área de Mecánica de Fluidos. Campus de Viesques, 33271, Gijón (Asturias), Spain.

jesusfo@uniovi.es

\begin{abstract}
Different sources of unsteadiness in low-speed axial turbomachinery are identified and classified in the present paper. From the classical picture segregating non-periodic mechanisms (turbulence) from periodic phenomena (unsteadiness), a further decomposition is outlined to distinguish between forced (deterministic periodicities) and unforced (non-deterministic) unsteadiness. Raw velocity traces, measured for several test conditions in a typical industrial fan with hot-wire anemometry, are ensemble-averaged to obtain statistically-stationary fluctuations. Then, a frequency-based filtering procedure is employed to isolate non-deterministic, but also non-chaotic, disturbances from such fluctuations, resulting in the so-called unforced unsteadiness. This term reveals coherent flow structures that involve "large-scale" unsteadiness with other periodic features different to the blade rotation scales (BPF). As a starting point, the kinetic energy associated to the total unsteadiness (in terms of a percentage of the kinetic energy of the time-averaged flow) is analyzed as a function of the operating conditions. Next, the different components contributing to the total unsteadiness of the flow are also observed, in order to determine their particular significance on the global unsteady scenario. It is shown that the turbulent kinetic energy reaches up to approximately a 50-60\% of the total unsteady energy, while both forced and unforced components contribute equally to the rest of the energy. In addition, it is observed that higher levels of unforced unsteadiness are concentrated towards the endwall boundary layers where forced unsteadiness is notably reduced due to the loss of the jet-wake structure. Conversely, forced unsteadiness is more evident at inner regions of the rotor passage. Furthermore, unforced unsteadiness is especially intense in the tip regions where large scales associated to the tip leakage vortex are established. It is demonstrated that the estimation of the unforced unsteadiness constitutes an accurate indicator of the presence of tip leakage flows for axial turbomachinery. Moreover, this is confirmed through the representation of the degree of anisotropy, where typical anisotropic structures are revealed. Finally, with the introduction of power spectrum densities for the unforced components, it is possible to identify typical eddy sizes of these large fluctuations.
\end{abstract}

\section{INTRODUCTION}

A complete description of the unsteady flow inside axial turbomachinery requires a deep insight into a wide range of different time and length scales. The flow structures involved present length and time scales varying within 5 or 6 orders of magnitude because the origin of these fluctuations is diverse [1]. Typically, turbulent mechanisms are inherently associated to small, dissipative scales, whereas organized flow patterns (jetwake structures, potential effects or streamwise vortices) are related to large scales in the order of a fraction of the machine diameter [2]. Hence, these opposite length scales represent the extremes of the whole fluctuation range (i.e. the total unsteadiness). Respectively known as "turbulence" and "unsteadiness" [3-4], they also stand for two different ways to describe high-complex, non-stationary flow fields: while turbulence is usually analysed with statistical schemes, deterministic unsteadiness is a well-expected, repeatable flow feature.

In recent years, the analysis of unsteadiness, particularly concerning blade row interaction, has become a major topic for research. It is well known that the work transfer inside a turbomachine takes place due to the unsteady flow induced by the tangential displacement of the blade-to-blade pressure gradients of the rotor ([5-6]). Moreover, the time-mean footprint of the unsteady processes in the time-averaged flow implies also important effects, especially involved in the performance and efficiency of the machine. Since energy exchange is established between the unsteady mechanisms and the time-averaged flow, further knowledge of these periodic events is extremely useful. Actual trends in design of axial turbomachinery take advantage of the assessment of these unsteady processes 
in both turbines and compressors, leading to important benefits and improvements [7-8].

On the other hand, turbulence is considered as a disturbing mechanism, modifying wake decay and interaction processes [9]. In fact, investigations of the levels of free-stream turbulence have shown the impact of dissipative scales on the unsteady transitional flow in both compressors [10-11] and turbines [12]. Furthermore, anisotropic turbulence associated to the shear layers of rotor wakes is an additional source of wake-induced unsteadiness leading to transition and generation of hot-spots [13]. As a result, detailed studies providing an accurate picture of coexisting turbulent and unsteady mechanisms are necessary.

Deterministic unsteadiness is obtained using an ensemble-averaging operator [14-15]. This technique provides the phase-averaged flow which includes all the unsteadiness related to the BPF. This deterministic flow, by definition, recovers all the time scales correlating only with the rotation of the machine [16]. Subtracting this processed data from the original traces, a statistically-stationary fluctuation is determined, mainly composed by turbulent structures. However, other "large-scale" unsteadiness with periodic features unrelated to the shaft speed is still present [17]. All these considerations are illustrated in the classification proposed in figure 1. The ensemble-averaged flow, denoted as "forced unsteadiness" includes all deterministic phenomena, either inviscid or viscous mechanisms. Complementarily, the remaining fluctuations are composed by turbulent structures and "unforced unsteadiness". Typically, this unforced unsteadiness consists in both stable and unstable mechanisms including vortex shedding, unsteadiness of separated points, misalignment of blades or tip and secondary vortices. This kind of large-scale unsteadiness is neither turbulence (this is not its nature) nor a strictly deterministic contribution. In fact, these phenomena are somewhere between the deterministic fluctuations and the chaotic turbulence.

Typically, most of the research has been focused either on deterministic unsteadiness or on turbulent structures. Unforced unsteadiness is usually ignored, introducing some kind of frequential methods that filter out its contribution. In particular, it is a common practice to introduce a complete elimination of certain ranges. Thus, some authors have defined a cut-off frequency to extract just "small-scale" fluctuations: Goto [17] introduced a high-pass filter of 1.5 times the BPF, while Senkter et al. [18-19] employed a filter of 0.2 times the BPF. Unfortunately, the drawback of this procedure is that much of the turbulent energy associated to large-scale eddies is neglected, resulting in an underestimation of the turbulent budget. Furthermore, all the unforced unsteadiness is thus completely ignored.

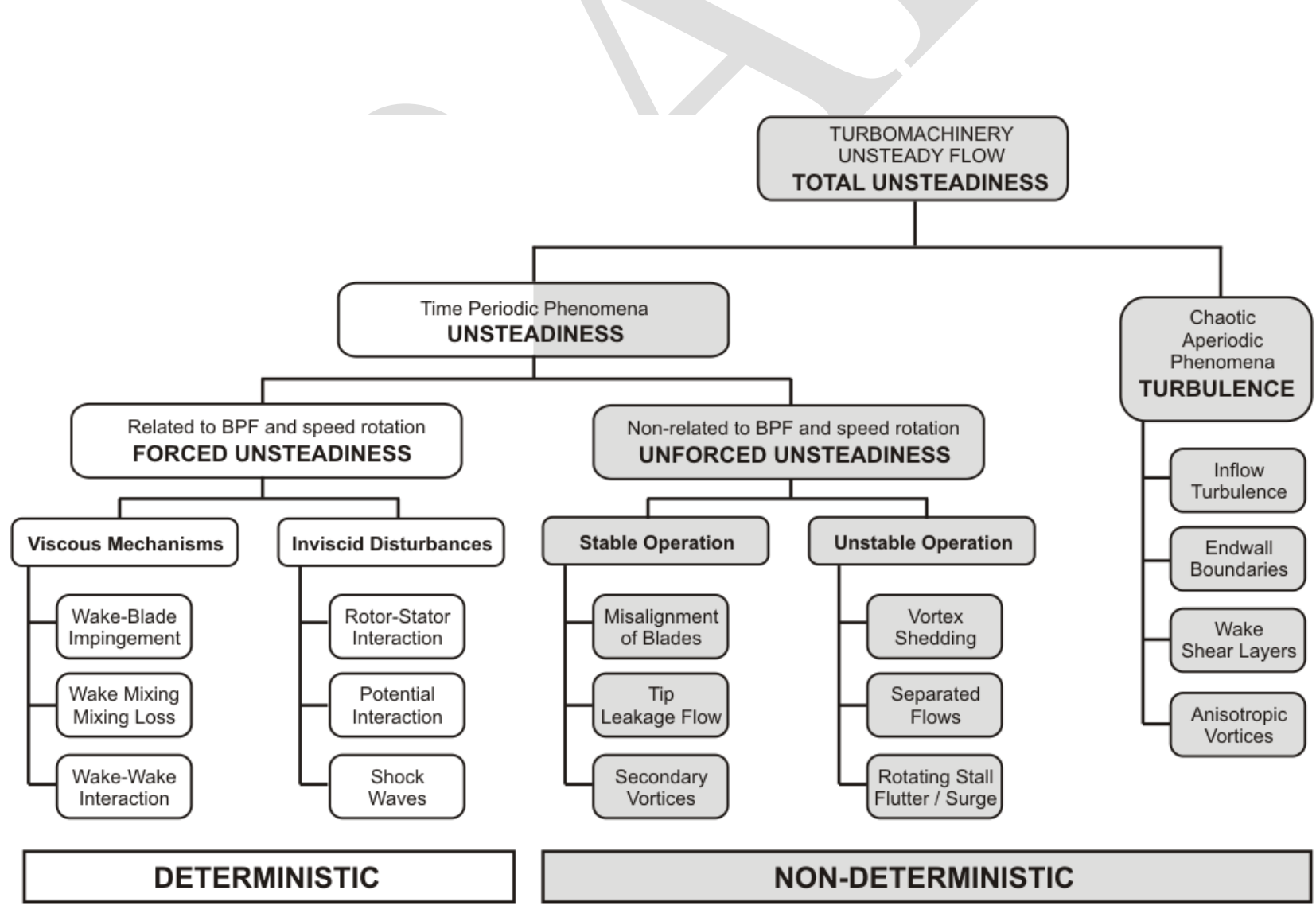

Fig. 1. Classification of unsteady processes in axial turbomachinery. 
In the present paper, the key point is the definition of an accurate method to isolate the unforced unsteadiness from the turbulent scales after phase-averaging. For that purpose, an enhanced frequential method is proposed to decouple unforced unsteadiness from pure turbulent structures. Previous investigations of the authors analysed the generation and transport of turbulent structures in axial turbomachinery [20-21], considering a filtering procedure to eliminate relevant peaks of the velocity spectra. Now, we revisit this methodology introducing a generalization that allows filtering out any periodic event in the spectra (not only higher harmonics of the BPF and the rotational frequency). It will be shown that the difference between original and truncated power spectra, when transformed back into the time domain, enables to determine maps of unforced unsteadiness in the rotor passages. In addition, it is demonstrated that higher levels of unforced unsteadiness are concentrated towards the endwall boundary layers, especially intense in the tip regions due to the establishment of the tip leakage vortex. Basically, the objective of the study is to advance a practical methodology to isolate unforced unsteadiness. Using experimental data, measured in a typical industrial fan with hot-wire anemometry, it is possible to derive some trends of the behaviour of the unforced unsteadiness with variations in operational parameters like the flow rate conditions or the blade pitch position.

\section{ON THE IDENTIFICATION OF UNFORCED UNSTEADINESS}

Time Scales. All the unsteady and turbulent phenomena in axial turbomachinery have been classified in figure 1 according to their periodic (or not) characteristics. Hence, total unsteadiness was segregated in three different parts, regarding forced and unforced unsteadiness and chaotic turbulence. Implicitly, it was assumed that small scales are associated to turbulent mechanisms while large scales are characteristic to both forced and unforced unsteadiness. Although this simplified idea of the time scales is a good approach to real life, it is necessary to introduce some additional considerations to enhance the big picture.

In particular, it is extremely important to notice that different unsteady mechanisms may contribute with fluctuations that are superimposed in narrow frequency ranges. This means that the unsteady energy content of particular frequencies can be contributed by two or more mechanisms. Furthermore, every unsteady flow structure produces disturbances throughout a singular interval, so it is expected that the complete collection of unsteady features inside a turbomachine derives in a complex, highly-interacted energy spectrum.

These ideas are evident for turbulent scales because the energy cascade in the inertial subrange reproduces a physical mechanism with such characteristics [22-23]. In the case of unsteadiness, this occurs when several mechanisms (forced or unforced) are manifested at similar scales. For instance, tip leakage vortex is a coherent flow structure, spatially associated to the blade tip clearance, which produces unforced unsteadiness at several length scales. This mechanism interacts with the passage flow structure, that is, with the pitchwise jetwake structure. As a result, the energy budget of the tip leakage flow is spared, mainly contributing as an unforced mechanism, but also transferring energy into the blade-to-blade deterministic flow pattern. The practical consequence is that a residual component of the tip vortex can be identified in the phase-averaged flow, though its major contents of energy show that the tip leakage flow has a definable distinct unsteadiness whose frequency is non-related to blade passing frequency [7]. In other words, the tip leakage vortex sheds a reduced part of its energy at the BPF rate, and as a consequence, it leaves a footprint in the unsteady ensemble-averaged fields. Although it has been classified as an unforced mechanism, it is welldocumented in the bibliography the identification of tip vortex structures within analysis of deterministic unsteadiness [24-26].

This high complex combination of time scales derives in the practical impossibility to isolate every particular unsteady (or turbulent) phenomenon. A more useful strategy is to be focused on the common characteristics of the mechanisms. Thus, we can analyze the three parts defined in the previous classification and provide a quantitative estimation of their relative importance. Even so, notice that the difference between forced and unforced unsteadiness is a very diffuse frontier. This is because the definition of the ensembleaveraging operator considers that such a frontier can be established at the BPF wavenumber. Thus, unsteady mechanisms contributing with energy in a frequency range including the BPF (i.e., the tip leakage flow) will be split between both types of unsteadiness. Although this is the drawback of the present classification, it is very important to notice that it is necessary to introduce a separating criterion between forced and unforced unsteadiness. Otherwise, energy of large-scale fluctuations is masked as large-scale turbulence, and periodic phenomena unrelated to the BPF cannot be distinguished from turbulent structures.

Before we introduce a practical method to segregate unforced unsteadiness from turbulence, it is worth it to illustrate the complex superimposition of time scales in the flow and how we can group relevant mechanisms according to the classification defined in figure 1. For that purpose, a representation of the power spectrum density of a velocity trace measured rotor downstream of an axial fan is included in figure 2. Over a logarithmically scaled $\mathrm{x}$-axis ( $\mathrm{y}$-axis is linearly scaled), it is represented the one-dimensional energy spectrum of the autocorrelation function. Typical scales (frequency ranges) corresponding to forced and unforced unsteadiness as well as turbulence have been indicated in the plot. Turbulence is clearly associated to the smallest length scales (higher frequencies). The energy budget of turbulent phenomena, including freestream, generation at endwall boundary layers and wake-induced turbulence, presents a classical broadband distribution. Just the inertial subrange has been pointed out in the figure, but it is well known that turbulent structures in one-dimensional spectra also contribute in 
low frequency regions [27]. In spectral space, a random array of eddies of fixed size tends to produce a fairly broad one-dimensional spectrum [23], progressively peaked towards $\mathrm{k}=0$. Then, much of the energy content at low frequencies is a consequence of the redistribution of turbulent energy in a one-dimensional spectrum. Notice that this may lead to notable underestimations in the levels of turbulence intensities when a cut-off frequency is employed to segregate turbulent phenomena from deterministic scales. In addition, this energetic redistribution implies that, in the region identified as typical scales of unforced unsteadiness, the background oscillations are clearly turbulent, while unforced mechanisms must be manifested as superimposed, discrete peaks. Hence, tip leakage vortex (if measuring close to the tip clearance), secondary vortex or wake oscillations are periodic events, so it is expected to observe periodicities (peaks in the spectrum) at relatively low scales. Finally, forced unsteadiness is perfectly identifiable at BPF and its harmonics. This kind of unsteadiness is strictly associated to a particular time scale, so it contains very important flow patterns, but with a moderate energy budget.

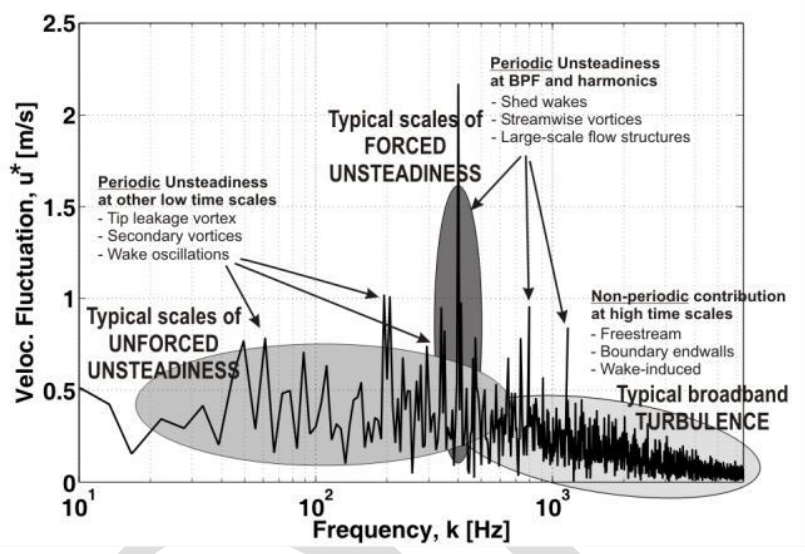

Fig. 2. Typical scales of forced, unforced and turbulent mechanisms.
In summary, typical scales associated to both forces and unforced unsteadiness and to turbulence have been illustrated. Though it is difficult to establish a perfect segregation of the phenomena or to draw clear limits between these three regimes, an attempt was made to link a classification based on the periodic features of the unsteady mechanisms with basic ideas about their length scales. A classical classification [28] divides the total unsteadiness in three regions: "small scales" involving wake and boundary layer turbulences, "medium scales" associated to passage flow structures like wake velocity patterns and tip vortices and "large scales" including flutter, rotating stall or surge. In some sense, the sketch in figure 2 situates the three parts of total unsteadiness defined in fig. 1 in that three zones of the overall frequency range, thus proposing a certain analogy between scales and types of unsteadiness. Thus, unforced unsteadiness is distributed over medium and large scales (see fig. 1), with unstable mechanisms more related to larger scales and stable phenomena in the order of the chord length.

Processing technique: a filtering procedure. We have already organized in a coherent manner the diverse array of unsteady flow events existing in axial turbomachinery. Following, it is time to introduce a procedure to segregate unforced unsteadiness from turbulent structures. For that purpose, it is assumed that we have at our disposal an experimental dataset composed of instantaneous velocity traces measured in an axial stage environment with hot wire anemometry. The measurements are taken in a traverse sector, with accurate sampling rates and long acquiring times (during several rotor turns). In addition, a signal from a shaft encoder must be used for synchronizing the time series with the phase of the machine. Thus, it is possible to identify every rotor phase when phase-averaging in the post-processing.

The first step is to segregate deterministic structures from original raw data. The velocity traces must be processed using the ensemble-averaging technique, so the phase-averaged is recovered. This is expressed as:
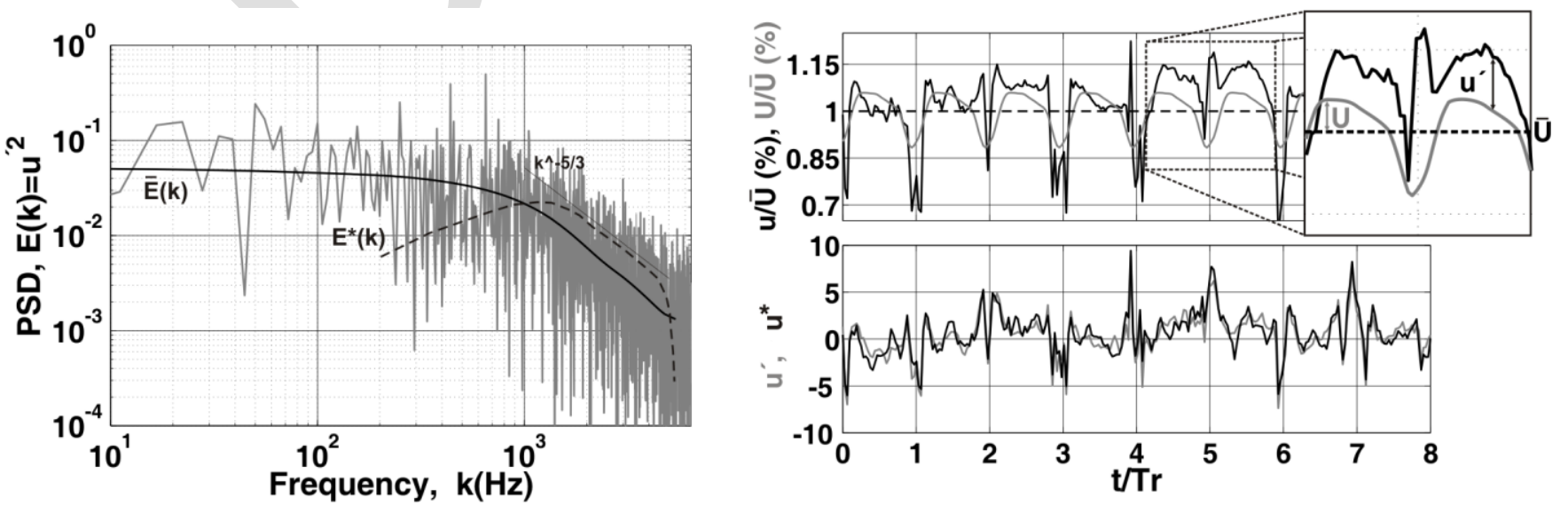

Fig. 3. Ensemble-averaging and turbulence spectrum. 


$$
\mathscr{U}(\stackrel{\mathrm{r}}{r}, t)=\left.\frac{1}{M} \sum_{m=1}^{M} u(\stackrel{\mathrm{r}}{r}, T)\right|_{m} \quad ; \quad T=t+\frac{2 \pi}{\Omega B}(m-1)
$$

where the variable $\stackrel{r}{r}$ represents the spatial coordinates and $t$ defines each of the instantaneous snapshots defined over the rotor blade passing period $\left(T_{r}\right) . B$ is the number of blades, $\Omega$ is the turbomachine rotational speed and $T$ computes the total acquiring time.

The non-deterministic fluctuations are obtained as the difference between the ensemble-average value and the instantaneous value:

$$
\left.u^{\prime}(\stackrel{\mathrm{r}}{r}, T)=u(\stackrel{\mathrm{r}}{r}, T)-\mathcal{Q}^{\stackrel{\mathrm{r}}{r}, t}, t\right)
$$

Figure 3 shows in the right column (top) a sample signal that will be used to illustrate the methodology employed to segregate deterministic and nondeterministic parameters. All the velocity components have been made non-dimensional with the overall mean value, $\bar{U}$. The gray line represents the phase-averaged flow where $U$ is the fluctuation associated to the deterministic scales. Notice that the jet-wake structure is perfectly recovered with the ensemble-averaging operator (replotted eight times over the instantaneous velocity deficits of the signal). The difference between the original data and the forced unsteadiness is $u^{\prime}$ which has been plotted below (gray line). This random component presents notable fluctuations, centred on zero. This is a direct consequence of having removed the unsteady deterministic component from the original series, so the remaining fluctuation is a statisticallystationary signal.

This sample trace has been reproduced here from experimental measurements in an eight-blade, lowspeed axial fan, rotating at $3000 \mathrm{rpm}$. More details about this particular turbomachine will be referenced later. However, further description of the phaseaveraged flow is out of the scope of the present paper. Complete analyses of the deterministic structures for this type of industrial axial were already carried out in previous investigations by the authors [29-30]. In the present section, just general comments concerning methodological aspects of the segregation of unforced unsteadiness are addressed over these sample traces.

In the left column of figure 3 we have represented the power spectrum density of the random trace, $u^{\prime}$ (gray spectrum). Both frequency and power axis are plotted in a logarithmic scale. The energy content of the non-deterministic scales is determined taking the Discrete Fourier Transform (DFT) of the time signal. Due to the mathematical restrictions followed by the FFT algorithm and inherent inaccuracies in the measurement (turbulence below the frequency response of the sensors is lost), there is a notable experimental scatter in the spectrum. The presence of those spurious spikes is typically mitigated smoothing the power spectra (averaging several spectra for every frequency [31]). Alternatively, in order to obtain a derivable function, we have employed a smoothing spline based on a moving average function that is defined along the whole frequency range, for a typical span of a $10 \%$ of the total points. In particular, each smoothed value is obtained with a locally weighted linear regression that uses a linear polynomial within that span. In addition to the regression weight function, a robust algorithm is employed to make the process resistant to outliers. The result is shown in the figure, superimposed to the original power spectrum (solid black line, $\bar{E}(k)$ ). As expected, this mathematically smooth spectrum exhibits the universal power-law decay in the inertial subrange (classical $-5 / 3$ exponential decay of nearly isotropic, fully-developed turbulence). Moreover, it is possible to estimate the three-dimensional spectra, $E^{*}(k)$, from its relation with the autocorrelation function according to [23]:

$$
E^{*}(k)=k^{3} \frac{d}{d k}\left[\frac{1}{k} \frac{d \bar{E}}{d k}\right]
$$

Notice that the smoothed spectrum has been obtained neglecting the contribution of those peaks that are clearly outside of the scattered amplitudes. Thus, both one-dimensional and three-dimensional spectra provide an accurate estimation of the energy content of the turbulent fluctuations in the inertial subrange. Therefore, evaluating the area under the smoothed spectrum, it yields:

$$
\frac{1}{2}\left\langle u^{\prime 2}\right\rangle=\int_{0}^{\infty} \bar{E}(k) d k
$$

Actually, both smoothed and original spectra must present exactly the same energy content because of the inherent randomness of the spurious spikes. Hence, we can apply equation (4) over the two spectra indistinctly, checking that the process mean has been accurately determined if equal values are obtained.

In the original spectrum, the passage flow scales were previously removed in the averaging sequence, so there are no relevant peaks at the blade passing frequency $(400 \mathrm{~Hz}$ in this particular case). But other periodic events could be still masked, embedded in the sea of random, scattered fluctuations. However, it seems quite problematic the discrimination between real periodic contributions and artificial spurious spikes from the representation of the figure. In any case, the experimental scatter is somehow statistically distributed over the smooth spectrum, pointing out that it is possible to estimate a statistical dispersion of the spurious data. Measuring how widely spread they are, we can define a statistical criterion to identify all those peaks falling outside some statistical control limits. Obviously, such out-of-range peaks represent the power spectrum of the unforced unsteadiness.

Subtracting the smoothed spectrum from the original one, a representation of the spurious values (including unforced unsteadiness) is plotted in figure 4 in a semilogarithmic scale for the $\mathrm{x}$-axis. A local quadratic error is calculated for every frequency in the 
representation, which is further smoothed using an additional locally weighted linear regression resistant to outliers. This mathematical construction allows us to define a moving standard deviation. Next, a confidence level is introduced considering that the control limits are fixed within two standard deviations. Obviously, all the amplitudes outside those boundaries are not considered as scattered data. Instead, these peaks falling beyond the specification are truncated in order to define the power spectrum of the unforced unsteadiness. Notice that the lower limit $(-2 \sigma)$ follows perfectly the inferior threshold of the spurious data. On the contrary, the upper limit is clearly segregating non-deterministic, periodic unsteadiness from the scattered randomness.

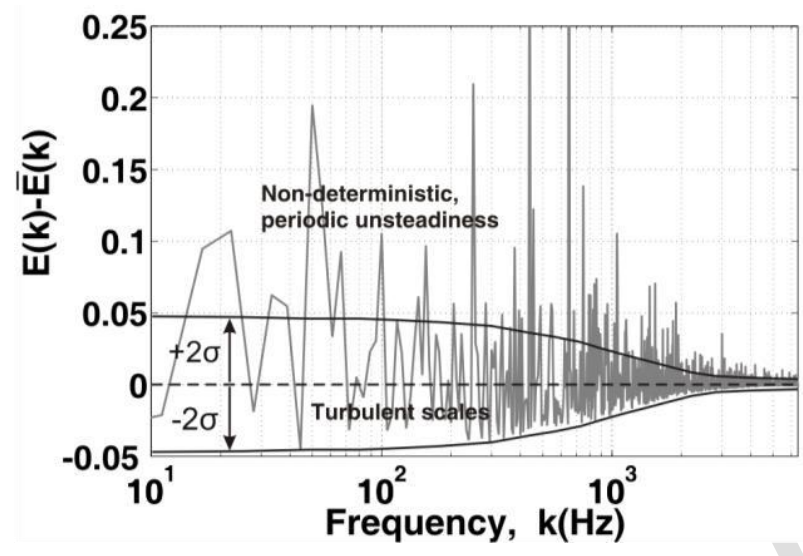

Fig. 4. Discrimination of unforced unsteadiness from turbulent scales.

Once the unforced unsteadiness is completely removed from the turbulent scales, the different parts of the total unsteadiness are represented separately in the power spectrum of figure 5 . The forced unsteadiness presents only periodic events at the BPF. The unforced component is composed of several periodic contributions, mainly distributed at low frequency ranges (below $1 \mathrm{kHz}$ in this sample), or medium-large scales. Also, notice that there are determined intervals where the unforced unsteadiness is not developed.

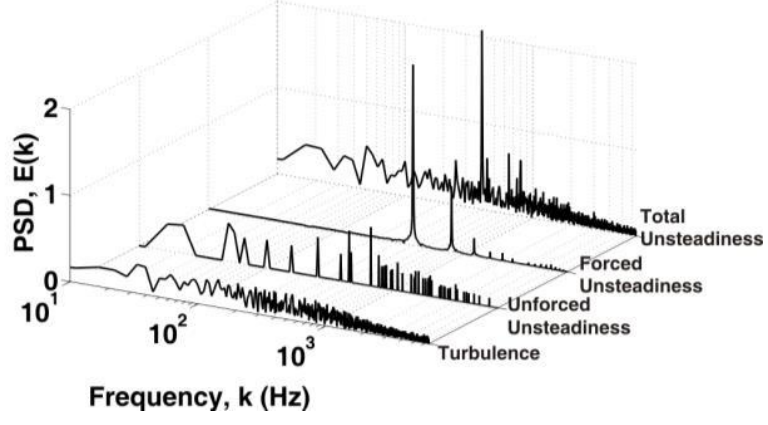

Fig. 5. Power spectrum of forced and unforced unsteadiness and turbulence
Finally, the turbulent structures show a broadband distribution over the whole frequency range, completely cleaned off any periodic feature.

All these components are then transformed back into the time domain in the final step of the segregating procedure. This inverse transformation is executed scaling the amplitudes of the raw data and then coupling these values with the phase information of every wavenumber coming from the original series. At this point, it is of special interest the comparison of the time series between the turbulent signals recovered from both smoothed and filtered spectra (figure 3, bottom at the right). The unique difference between them is the elimination of the scattered values. Note that this derives in minor, almost negligible differences between them.

\section{AXIAL TURBOMACHINERY: TEST CASE}

Research facility. This methodology has been applied over a low-speed axial fan with variable pitch blades to analyze the different characteristics of the unforced unsteadiness. The turbomachine was operated in an open-loop facility that includes a throttle at the facility discharge to provide off-design conditions. The fan analyzed is an eight-blade rotor with hub and tip diameters of 380 and $600 \mathrm{~mm}$ respectively (Fig. 6). The rotor, whose blade pitch angle can be manually changed, rotates at $3000 \mathrm{rpm}$ and is driven by a DC 36 $\mathrm{kW}$ motor.

Hot wire measurements were accomplished for 12 different cases: four blade pitch angles running at 3 different operating conditions. These pitch angles correspond to blade angles measured at the hub section with respect to the tangential direction of 43.5, 48, 52.5 (design) and $57 \mathrm{deg}$; from now on, referred as “-9 $9^{\circ}$ ", “$4.5^{\circ}$ ", " 0 " " and " $+4.5^{\circ}$ ". In addition, the three operating points measured the best efficiency point $\left(Q_{o}\right)$ for each pitch angle and a lower and a higher flow rate: $0.95 x Q_{o}$ and $1.05 x Q_{o}$. Information related to the facility configuration, rotor geometries and performance and operation of the axial fan can be found in [21].

Measurement technology. Tri-axial hot wire anemometry techniques were employed to measure the three velocity components rotor downstream. The hot wire probe is composed of three tungsten filaments ( 5 $\mu \mathrm{m}$ dia.), orthogonal to each other, forming a regular quadrant in space with a $30 \mathrm{deg}$ inclination with respect to a plane normal to the prongs. The probe, connected to TSI IFA-100 CTA anemometer, has been used to measure downstream the exit of the blade row, over 10 radial positions clustered towards the endwall. More details about typical frequency response and the uncertainty of the probe as well as the calibration setup are summarized in [32].

Data from the hot wire probe was sampled at 12.8 $\mathrm{kHz}$ per channel, corresponding to 32 samples each blade passing period, in order to obtain a good resolution of the jet-wake structure. Finally, phase locking averaging data procedure is done over 10 rotor revolutions. 


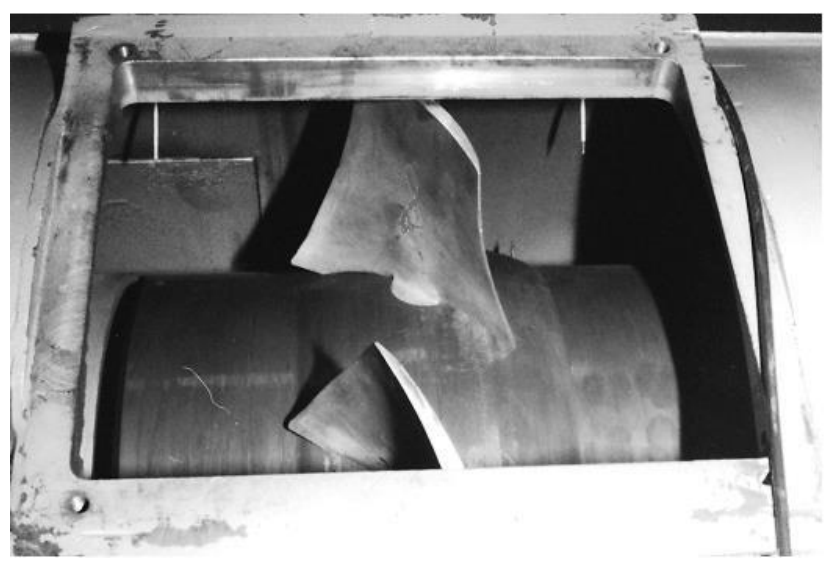

\begin{tabular}{|c|c|c|c|}
\hline & Tip & Midspan & Hub \\
\hline $\begin{array}{l}\text { Diameter [mm] } \\
\text { Number of blades }\end{array}$ & 380 & $\begin{array}{c}490 \\
8\end{array}$ & 600 \\
\hline Chord [mm] & 144.9 & 149.7 & 156.9 \\
\hline Blade pitch angle [deg] & 27.4 & 33.2 & 52.5 \\
\hline Camber angle [deg] & 18.6 & 22.5 & 42.9 \\
\hline Solidity $[-]$ & 0.615 & 0.778 & 1.052 \\
\hline Hub-to-tip ratio $[-]$ & & 0.63 & \\
\hline Tip clearance $[-]$ & & $2 \%$ span & \\
\hline Reynolds number [-] & & 6xE05 & \\
\hline Design Pressure Rise [-] & & 0.337 & \\
\hline Design Flow Coeff. [-] & & 0.35 & \\
\hline
\end{tabular}

Fig. 6. Rotor characteristics

\section{RESULTS AND DISCUSSION}

This final section is focused on the analysis of the unforced deterministic scales of the flow. Particular attention is paid on the relative importance of this part on the total unsteadiness of typical axial flow fans. Then, as a starting point, main characteristics of the kinetic energy associated to total unsteadiness in this type of turbomachinery is revisited, summarizing basic trends discussed in previous investigations [21].

Total unsteadiness. Variable pitch blades are usually employed in industrial, low-speed fans as an economic solution to design axial machinery that has to be operated delivering variable flow rates. In general, they present a limited range of operational conditions where the efficiency is not excessively compromised. However, it is well known that progressive reductions of the blade pitch angle produces important geometrical blockages in the passages, leading to more constricted flows and severe increments of the instabilities in the flow structures. Furthermore, most of these industrial fans are designed to be as compact as possible, introducing important camber angles and reduced spans. This gives rise to high aerodynamic loads and, as a consequence, large differences between pressure and suction side conditions. These conditions support a pronounced jet-wake structure and reinforce the tip leakage flow, inducing important overall levels of unsteady kinetic energy.

Figure 7 shows the radial distribution of total unsteadiness in this axial fan and how it is modified according to changes in the pitch angle or the operating conditions. The total unsteadiness, calculated as the difference between the instantaneous velocity and its time mean value, is then transformed using a DFT algorithm to obtain its power spectrum. Evaluating the area under such spectrum, the kinetic energy associated to the total unsteadiness is computed. This value is compared to the kinetic energy of the time mean flow, i.e. the kinetic energy of the bulk flow.

Significant values of $20-30 \%$ are found in the central positions of the rotor span. At midspan, the major contribution is due to the periodic unsteadiness of the wake fluid and the anisotropic turbulence generated in the shear layers of the velocity deficit [30]. On the other hand, towards the endwalls, the energy associated to total unsteadiness is severely increased, reaching up to levels beyond $100 \%$. This increment is a consequence of the small-scale turbulence developed inside the casing viscous regions. Moreover, maximum levels are even higher in the tip region, rather than in the hub section, due to the contribution of the tip vortex. Notice that these radial trends are shared by all the operating conditions and pitch angles, though running at off-design conditions and with reduced pitch blade angles increase the total levels of disturbance.
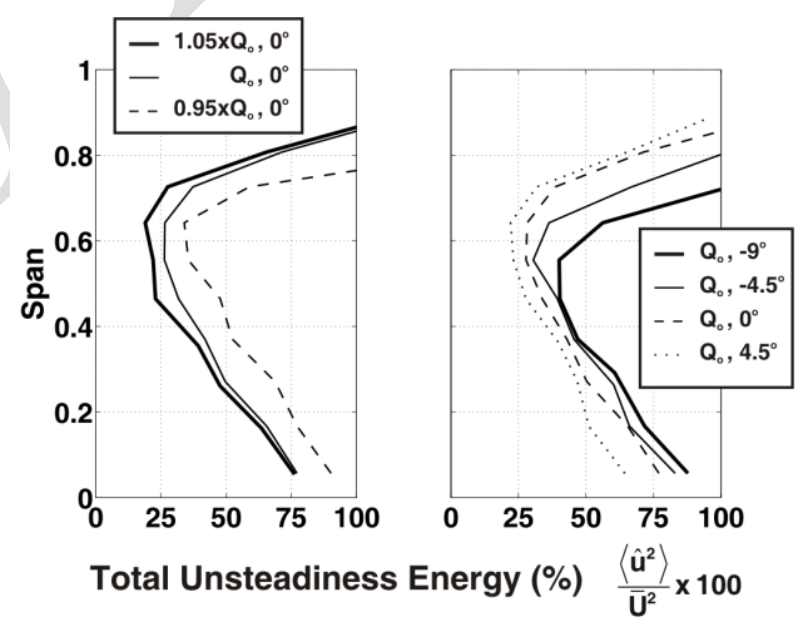

Fig. 7. Radial distribution of total unsteadiness energy as a function of operating parameters.

To summarize the effect of operational parameters, figure 8 has been included here, adapted from [21] but now in terms of percentage of the mean kinetic energy. This diagram represents interpolated levels of unsteady energy as a function of both parameters at misdpan. It shows that the lower the blade pitch angle, the higher the levels of total unsteadiness. Also, reduced flow rates lead to major angles of incidence (less margin to prevent stall is available), and therefore, major flow separation may occur near the trailing edge resulting in a significant level of disorder at the rotor exit. 


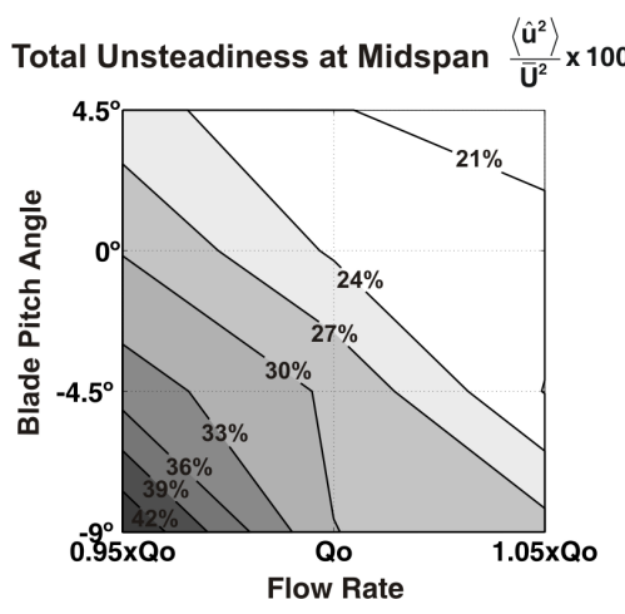

Fig. 8. Diagram of total unsteadiness energy at midspan. Influence of operating conditions.

Unforced unsteadiness. The radial distribution of the different components contributing to the total unsteadiness is presented in figure 9. These results correspond to the best efficiency point of the design blade angle, though similar global trends are observed for the other operating conditions (not shown here).

The comparison is carried out in terms of the kinetic energy associated to each component. This metric is particularly suitable because it enhances a perfect segregation among them addressing their relative importance. Recall that these energies are calculated as the area under the power spectrum density of the velocity magnitude for each component (fig. 5).

The representation is a bar graph of the total unsteadiness (i.e. its associated unsteady kinetic energy) which includes the relative contribution (in percentage) of each component at every radial position. For every bar, the black part represents the turbulent kinetic energy whereas white and grey parts correspond to the forced and unforced components respectively.

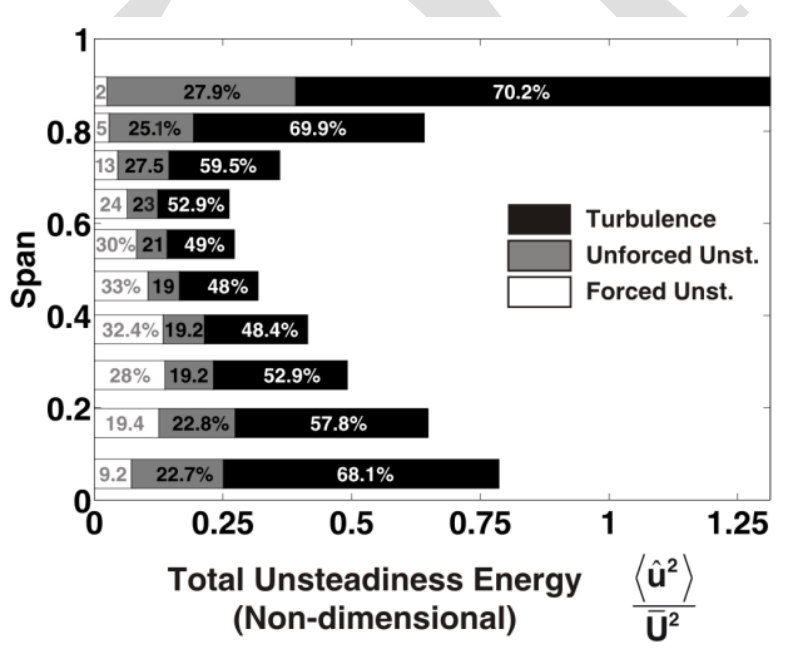

Fig. 9. Radial distribution of all the unsteady components (design conditions).
As expected, there is a very important spanwise contribution of the turbulent scales in the unsteady flow. Approximately, $50 \%$ of the kinetic energy associated to the total unsteadiness derives from turbulent mechanisms. This means that turbulence shed by the rotor wakes is a major inducer of flow disturbances. At midspan, the mixing out process of deterministic structures contributes to enlarge the turbulent fluctuations. Also, notice that towards tip and hub boundary layers, the weight of this component is increased (up to $70 \%$ ) due to the lost of the jet-wake structure.

Complementarily, forced and unforced components balance one to each other along the radial direction. Deterministic patterns are predominant at midspan (up to $30 \%$ of the total), though it is also observed a significant unforced unsteadiness (in the order of a 20\%). This unforced phenomenon has to be mainly related to wake oscillations and vortex shedding of separated flows generated at the trailing edge of the blades. As we move towards the endwall boundary layer, the unforced unsteadiness is progressively increased, especially reinforced in the tip region due to the influence of the tip vortex. Note that in those positions, deterministic energy is practically vanished.

Following, we analyze the effect of the operating conditions over the evolution of the different components of the total unsteadiness. Figure 10 shows a similar diagram to the one explained in figure 8 , but now for all the unsteady components. Important conclusions can be drawn from this figure. First, the trend followed by the forced component (left map) is completely opposite to the behaviour of the total unsteadiness shown in figure 8 . In this case, higher flow rates and higher pitch angles contribute to an increment of the wake deficit. The streamwise flow is increased, so the velocity gradient of the wake fluid is enlarged and higher fluctuations of the jet-wake structure overcome. Secondly, the turbulent contribution (right map) exhibits a quite independent behaviour with respect to flow rate. In this case, the key parameter is the generation of turbulence associated to the rotor blockage when the pitch angle is progressively reduced. Notice that both turbulent and forced mechanisms show important quantitative variations with the operating conditions. Thirdly, the unforced component (central map) presents moderate variations, from 18 to $24 \%$, which indicates a relative significance of this component at midspan locations. Moreover, this component is practically independent to variations in the pitch angle, but exhibiting a certain influence by the flow rate conditions. At central positions of the rotor passage, the unforced unsteadiness that can be developed is mainly due to wake oscillations and vortex shedding (separated flows) in the case of incompressible flow. Since reduced flow rates are responsible for wider rotor wakes, this could also be traduced in higher instabilities of the separation points in the blades surfaces, and thus higher levels of unforced unsteadiness. 

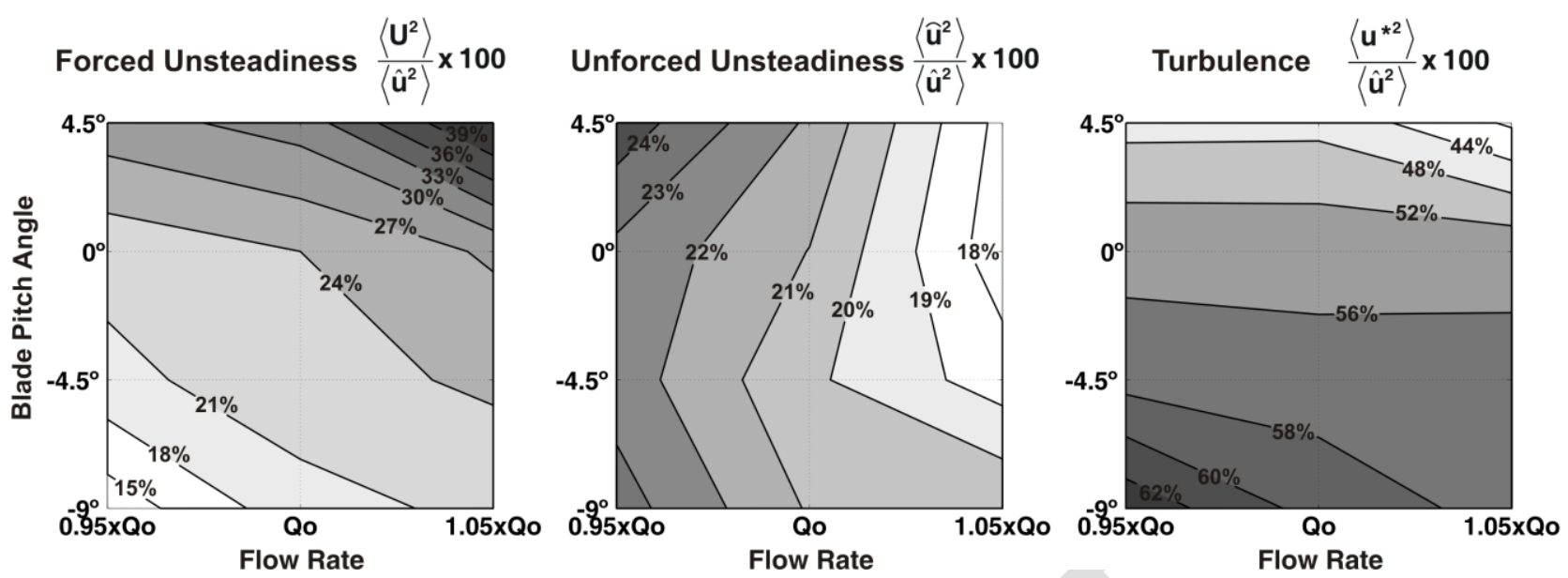

Fig. 10. Diagram of all the components energy at midspan. Influence of operating conditions.

Phase-averaged unforced unsteadiness. The frequential segregation of the unforced component allows us to obtain a "fictitious" temporal series when its power spectrum (fig. 5) is transformed back into the time domain. This component, as well as the turbulent component or the total unsteadiness, can be further ensemble-averaged to get valuable information about the transport of these unsteady structures in the deterministic flow patterns. Figure 11 shows the results obtained for two different operating conditions (other cases present similar features): in the left column, it is represented a low flow rate with a much reduced pitch angle; in the right column, results for a high flow rate with a reduced pitch angle are illustrated. The kinetic energy associated to every component is made nondimensional with the kinetic energy of the bulk flow as usual. Since these blade-to-blade maps are drawn from temporal ensembles, the phase-averaged kinetic energy is calculated as the sum of the three components to the square, that is:

$$
\left.k_{T}=\frac{1}{2} \underset{\left(\hat{u}_{i}\right.}{2} \hat{u}_{i}\right) ; k_{t}=\frac{1}{2}\left(u_{i}^{*} u_{i}^{*}\right) ; k_{u}=\frac{1}{2}\left(\vec{u}_{i} u_{i}\right)
$$

The whole spatial distribution of the variables (radial and tangential) is shown covering the rotor passage (replotted twice), viewed in the downstream direction. As expected, both total unsteadiness and turbulence present two clear flow patterns with generation of unsteadiness: the endwall boundary layers and the rotor wakes. Since the major contributor to the total unsteadiness is the turbulent component (up to a $50 \%$, previously discussed), it is presumable that both distributions reveal analogous maps, with a lowintensity core limited by the rotor wakes and hub and tip boundary layers with higher levels. Previous results [21] demonstrated that the progressive reduction of the pitch angle derives in an enlargement of the tip boundary layer and the dispersion of the rotor wakes, whereas higher flow rates tend to control these two disturbances. In the present figure, we have tried to illustrate these two opposite situations.
Now we focus on the distribution of phase-averaged unforced unsteadiness (bottom maps). In those maps, the rotor wakes are weft printed, so it is possible to recognize both pressure (PS) and suction sides (SS). However, in the distribution of unforced energy, the rotor wakes are hardly perceptible. On the contrary, an intense core of unsteadiness is clearly visible between the rotor wakes in the tip region, which indicates the contribution of the tip vortex structures onto the unforced component.

Moreover, as the casing boundary layer is enlarged due to the regulation (decrease) of the flow rate by means of a lower pitch angle (flow blockage), the leakage flow is progressively shifted towards the midspan. Similar conclusions were obtained analyzing helicity maps over the deterministic structures in previous investigations. Now, we confirm these overall trends over the energy budget associated to nondeterministic scales. In addition, at the hub there is also a remarkable presence of unforced phenomena, which could indicate the development of passage vortices.

The representation of ensemble unforced unsteadiness has revealed some unsteady mechanisms, like the tip vortex, that are transported by the unsteady (deterministic) flow patterns. Introducing the phaseaveraging operator, a spatial link is established between these structures and its unsteady advection, so we can track how the unforced energy is mixed out. However, it is also of interest the analysis of the energy contents of the unforced spectrum because they may provide information about typical length scales. Considering the same operating points as before, figure 12 shows the radial distribution of the power spectrum density for the unforced component. This component, related to medium-large scales, shows a power spectrum that is practically negligible for frequency ranges beyond 2 $\mathrm{kHz}$. On the contrary, energetic wavenumbers are found in the range between 200 to $500 \mathrm{~Hz}$, clearly activated towards the casing regions. Tip leakage flow and secondary (passage) vortices were identified as typical contributors to the unforced unsteady map in figure 11 . Now, with the frequential representation, it is possible to estimate the largest scales associated to this 
phenomena. In particular, at tip regions, there is a notable increase of the unforced energy over an interval centered in the frequency of $300 \mathrm{~Hz}$; whereas hub zones present fluctuations around $500 \mathrm{~Hz}$. These relevant peaks in the spectrum illustrate which are the characteristic scales of tip vortex and secondary vortices.
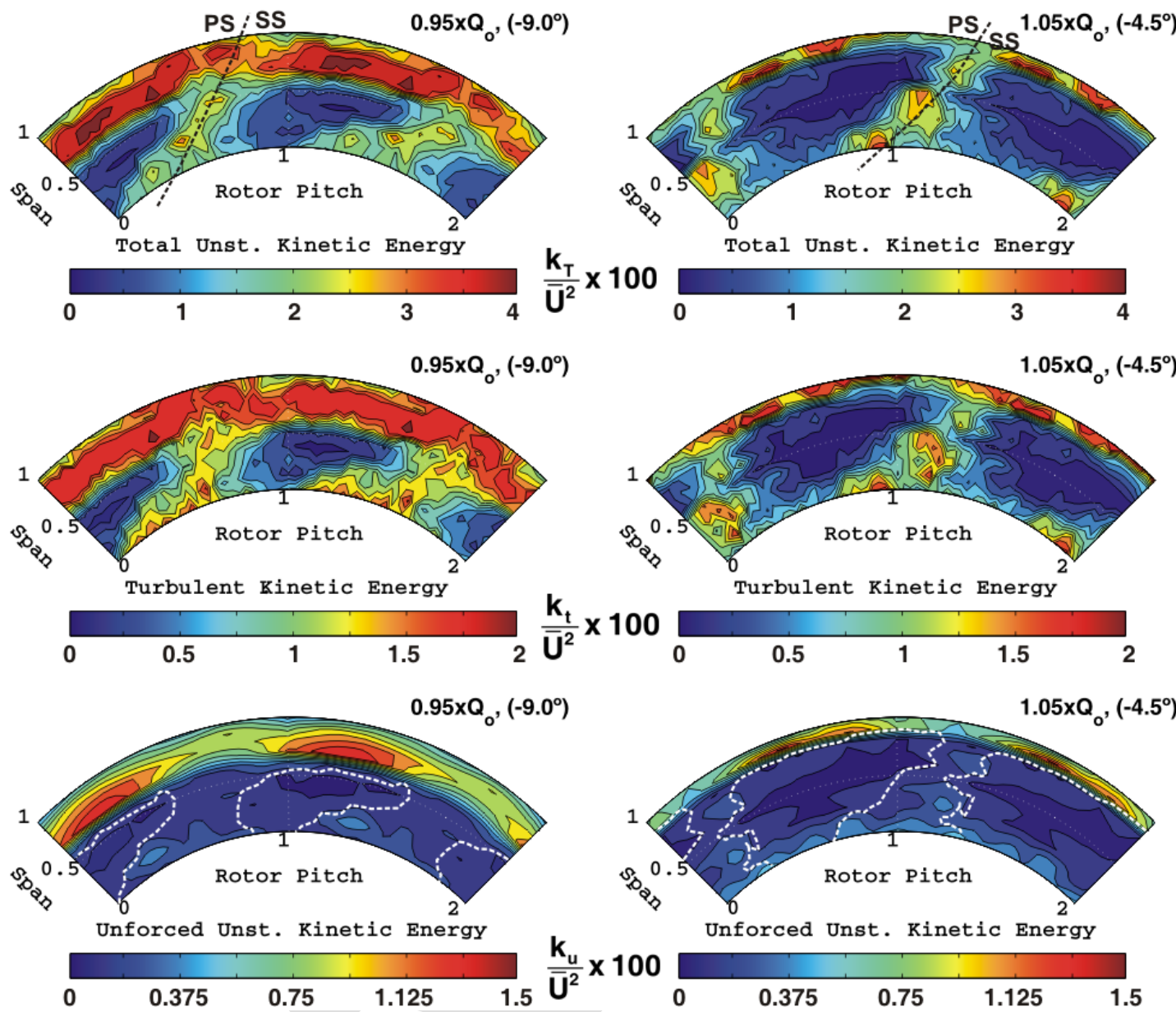

Fig. 11. Blade-to-blade distributions of turbulence and total and unforced unsteadiness for two different operating points.

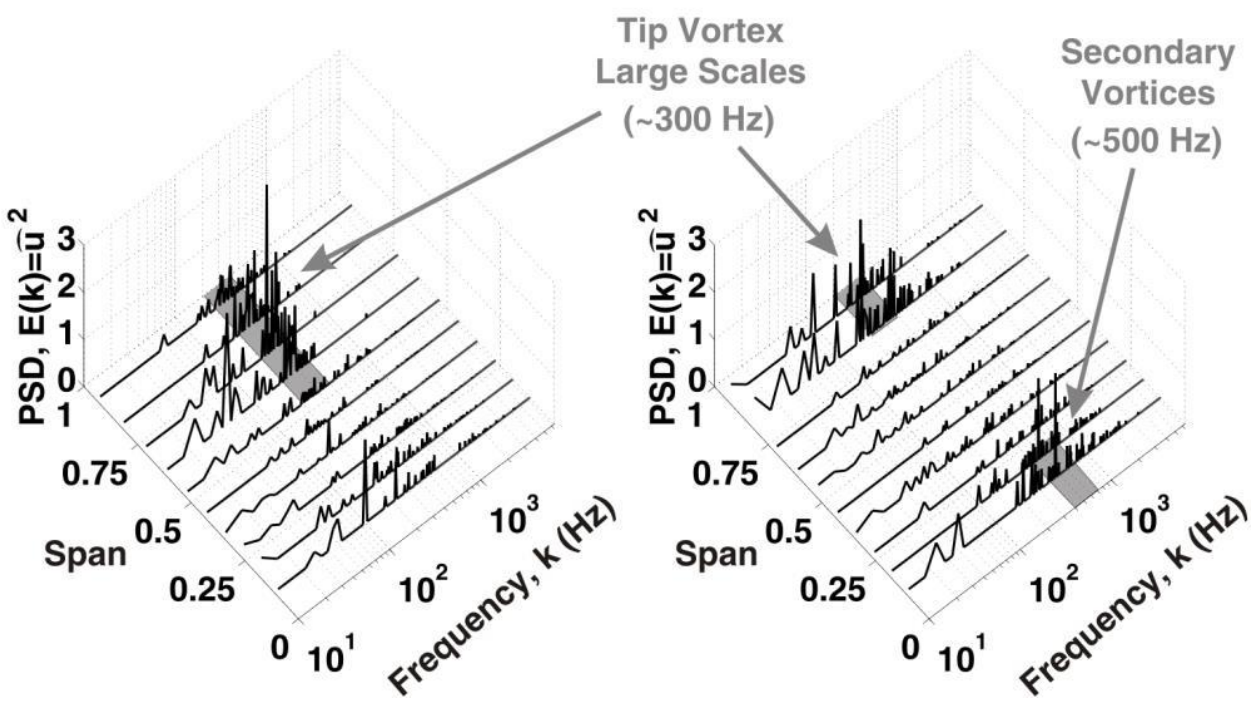

Fig. 12. Radial distribution of power spectrum density of unforced unsteadiness for two different operating points. 
Further insight is on the characteristic scales of unforced mechanisms is advanced within illustrative results in figure 13. This figure relates the phase-averaged distribution of the unforced unsteadiness with the relevant periodicities observed in their power spectra. In particular, spectra measured at tip, midspan and hub positions are shown in the figure. Results from the design conditions are recovered for this analysis. At $85 \%$ of the midspan, the contribution coming from the tip vortex is the clear source of unforced unsteadiness. As expected, the most energetic contributions in the spectrum for this component are observed for scales ranging around $300 \mathrm{~Hz}$. On the other hand, there is also an increase of the unforced component at hub locations, which in this type of axial fan was found to be at 500 $\mathrm{Hz}$ approximately. Finally, at midspan, there is a moderate unforced component, which could be associated to oscillations of the rotor wakes. In this case, a frequency value of $150 \mathrm{~Hz}$ appears to be the relevant periodicity of this mechanism.

Finally, the degree of anisotropy as defined in [33] is introduced to quantify the departures of isotropy of the flow structures associated to the unforced unsteadiness. It is expected to observe an important degree of anisotropy for the tip leakage vortex, due to its inherent level of vorticity. The degree of anisotropy is evaluated by comparing phase-averaged values of unforced unsteadiness according to:

$$
D A=\frac{2 \widehat{u}_{z}^{2}}{\widehat{u}_{\theta}^{2}+\widehat{u}_{r}^{2}}
$$

When this parameter is close to unity, the flow structure presents a clear isotropic characteristic. On the contrary, higher levels indicate anisotropic patterns. Figure 14 shows the results obtained for previous cases analyzed in figures 11 and 12. Except where the tip vortex structures have been identified, low levels of anisotropy are found in the rotor passage. On the contrary, tip vortex exhibits an important DA, with typical values around 4.

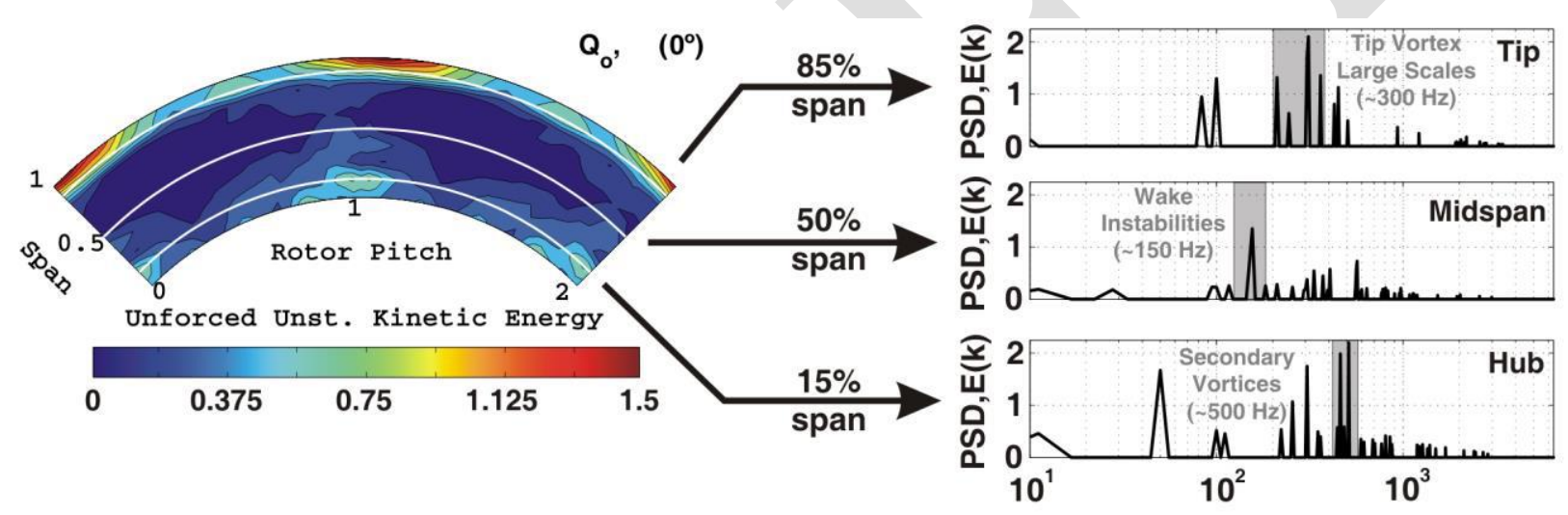

Fig. 13. Identification of typical time scales of unforced unsteady mechanisms at different radial positions.
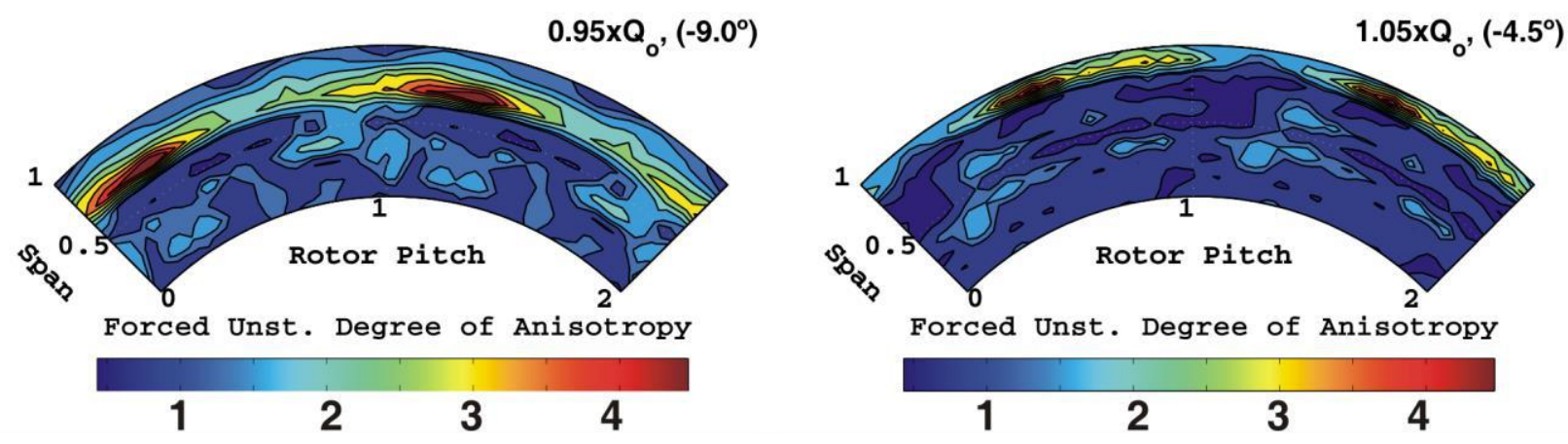

Fig. 14. Degree of anisotropy for unforced unsteady mechanisms for two different operating points.

\section{CONCLUSIONS}

In the present paper, a deep insight on the unsteady structures involved in low-speed axial turbomachinery is carried out. Particular attention is paid in the typical scales and periodic (or non-periodic) characteristics of the different unstedy mechanisms set off in the machine.
As a starting point, a classification based on the periodic nature of these phenomena is established, segregating the total unsteadiness in three different components: forced unsteadiness, unforced unsteadiness and turbulence.

Following, a frequential methodology is presented in order to differenciate unforced unsteadiness from pure turbulence. Firstly, a classical phase-averaging 
(ensemble) operator is necessary to obtain statisticallystationary fluctuations which are further post-processed to isolate the unforced component. The main goal of the investigation is the presentation of this unforced unsteadiness as a periodic (but non-related to the BPF) component, which energy content supports relevant flow structures like tip vortex or passage vortices. This component is also addressing all those large-scale energy associated to rotating stall, surge, misaligment of blades or wake oscillations (unstable separation points); otherwise masked as large-scale eddies of random turbulence.

Evaluating the unsteady kinetic energy of every component, it was possible to draw the contribution of the different parts onto the total unsteady energy. It was shown that for this type of industrial fans (flow rate regulation using variable pitch blades), major contribution onto the flow disturbances is due to the presence of turbulent structures (anisotropic shear layers of rotor wakes and casing boundary layers). Approximately, a $50 \%$ of the total unsteady energy stems from this component. In addition, both forced and unforced components were found to contribute with similar values to the total levels. Also, the unforced components was shown to be especially increased in the tip region, due to the contribution of the tip vortex and the redution of the forced component as a consequence of the loss of the jet-wake structure. In addition, the influence of operating conditions (flow rate and blade pitch angle) over the different unsteady parts was also analyzed at midspan, resulting in a certain independency of unforced unsteadiness to variations of the blade pitch angle (unstable separation points more related to changes in the flow rate)

Finally, phase-averaged maps of unforced unsteadiness were also determined. This representation allows us to observe the transport of the unforced phenomena on the deterministic unsteady patterns. The contribution of the tip leakage flow, and the presence of passage vortices has revealed the clear identification of these two coherent flow structures as unforced, nondeterministic unsteadiness. Introducing power spectra at different radial positions, it was possible to estimate the characteristic scales of all these phenomena. In particular, it was shown that wavenumbers centered on $300 \mathrm{~Hz}$ correspond to typical time scales of the largest eddies shed by the tip vortex, while higher time scales, around $500 \mathrm{~Hz}$, are identified with secondary hub vortices.

\section{ACKNOWLEDGEMENTS}

This work was supported by the Research Project "Characterization of the Generation of Aerodynamic Noise due to the Interaction between the Rotor Blades and the Guide Vanes in Axial Flow Fans", DPI200615720, CICYT.

\section{NOMENCLATURE}

$B=$ number of rotor blades

$\mathrm{BPF}=$ blade passing frequency, $\mathrm{s}^{-1}$

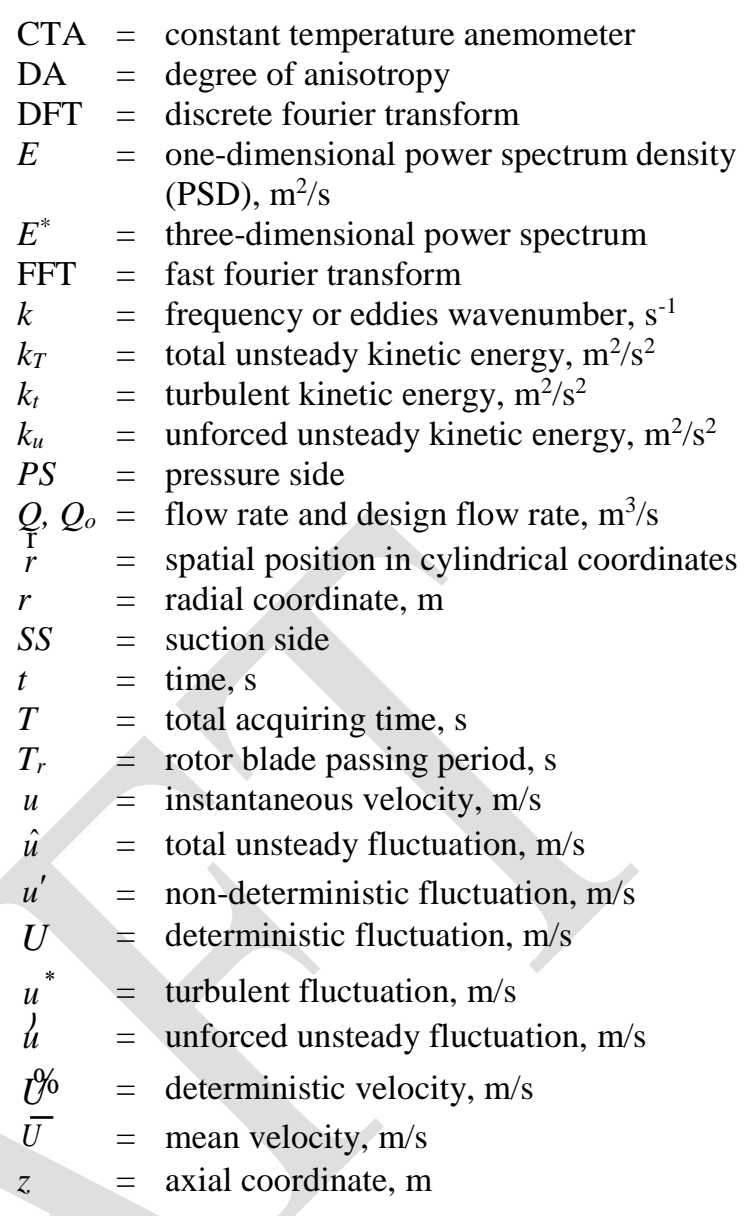

\section{Greek letters}

$\theta=$ angular coordinate, $\mathrm{rad}$

$\sigma=$ standard deviation

$\Omega \quad=$ rotational speed, $\mathrm{rad} / \mathrm{s}$

\section{Superscripts and Subscripts}

$$
\begin{aligned}
\langle\rangle & =\text { integral } \\
\% & =\text { ensemble-averaging } \\
-\quad & =\text { time-averaging } \\
i & =\text { component }
\end{aligned}
$$

\section{REFERENCES}

[1] R.S. Abhari, Unsteady fluid dynamics of turbines: A perspective on possible directions to improve future engine designs, Proceedings of Minnowbrook V, Workshop on Unsteady Flows in Turbomachinery, Blue Mountain Lake, New York, 20-23 August, 2006.

[2] J.J. Adamczyk, Modelling the effect of unsteady flows on the time average flow field of a blade row embedded in an axial flow multistage turbomachine, VKI Lecture Series on Unsteady Flows in Turbomachines, 1996-05.

[3] O.P. Sharma, G.M. Stetson, W.A. Danields, E.M. Greitzer, M.F. Blair, R.P. Dring, Impact of periodic unsteadiness on performance and heat load in axial flow turbomachines. Final Report NASA-CR-202319, NASA Lewis Research Center, Cleveland, Ohio, USA, 1997. 
[4] O. Uzol, Y-C. Chow, J. Katz, C. Meneveau. Experimental investigation of unsteady flow field within a two-stage axial turbomachine using particle image velocimetry. ASME Journal of Turbomachinery 124 (2002) 542-552.

[5] E.M. Greitzer, C.S. Tan, M.B. Graf, Internal Flow: Concepts and Applications, Cambridge University Press, 2004.

[6] S.L. Dixon, Fluid Mechanics and Thermodynamics of Turbomachinery, $4^{\text {th }}$ Ed. Butterworth-Heinenmann, 1998.

[7] C.S. Tan, Unsteady flows in compressors. VKI Lecture Series on Effects of Aerodynamic Unsteadiness in Axial Turbomachines, 2005-03.

[8] J.J. Adamczyk, Wake mixing in axial flow compressors, ASME Paper No. 96-GT-029, 1996.

[9] A.D. Henderson, G. Walker, J.D. Hughes, Unsteady Transition Phenomena at a Compressor Blade Leading Edge, ASME Paper GT2006-90641, TurboExpo, Barcelona, 8-11 May, 2006.

[10] A.D. Henderson, G. Walker, J.D. Hughes, The influence of turbulence on wake dispersion and blade row interaction in an axial compressor, ASME Journal of Turbomachinery 152 (2006) 150-157.

[11] R. Mailach, K. Vogeler, Aerodynamic blade row interactions in an axial compressor - Part I: unsteady boundary layer development, ASME Journal of Turbomachinery 126 (2004) 35-44.

[12] H.P. Hodson, R.J. Howell, Bladerow interactions, transition, and high-lift aerofoils in low-pressure turbines, Annual Review of Fluid Mechanics 37 (2005) 71-98.

[13] Y-C. Chow, O. Uzol, J. Katz, Flow nonuniformities and turbulent hot spots due to wake-blade and wake-wake interactions in a multi-stage turbomachine, ASME Journal of Turbomachinery 124 (2002) 553-563.

[14] B. Lakshminarayana, Techniques for aerodynamic and turbulence measurements in turbomachinery rotors, ASME Journal of Engineering and Power, 103 (1981), 374-392.

[15] C. Tropea, A.L. Yarin, J.F. Foss (Eds), Handbook of Experimental Fluid Mechanics, Springer, ${ }^{\text {st }}$ Ed, 2008.

[16] F. Leboeuf, Unsteady flow analysis in transonic turbine and compressor stages, VKI Lecture Series on Recent Developments in Numerical Methods for Turbomachinery Flows, 2002-01.

[17] A. Goto, Three-dimensional flow and mixing in an axial flow compressor with different rotor tip clearances, ASME Journal of Turbomachinery 114 (1992) 675-685.

[18] A. Senkter, W. Reiss, Measurement of unsteady flow and turbulence in a low speed axial compressor, Experimental Thermal and Fluid Science 17 (1998) 124-131.

[19] A. Senkter, W. Reiss, Experimental investigation of turbulent wake-blade interaction in axial compressors, International Journal of Heat and Fluid Flow 21 (2000) 285290.
[20] J.M. Fernández Oro, K.M. Argüelles Díaz, C. Santolaria Morros, E. Blanco Marigorta, On the structure of turbulence in a low-speed axial fan with inlet guide vanes, Experimental Thermal and Fluid Science 32 (2007) 316-331.

[21] J.M. Fernández Oro, R. Ballesteros-Tajadura, E. Blanco Marigorta, K.M. Argüelles Díaz, C. Santolaria Morros, Turbulence and secondary flows in an axial flow fan with variable pitch blades. ASME Journal of Fluids Engineering 130 (2008) - Accepted for publication.

[22] H. Tennekes, J.L. Lumley, A First Course in Turbulence, The MIT Press, 1972.

[23] P.A. Davidson, Turbulence: An Introduction for Scientists and Engineers, Oxford University Press, 2004.

[24] J. Bae, K. Breuer, C.S. Tan, Periodic unsteadiness in compressor tip clearance vortex, ASME-GT-53015

[25] C.-M. Jang, D. Sato, T. Fukano, Experimental analysis on tip leakage and wake flow in an axial flow fan according to flow rates, ASME Journal of Turbomachinery 127 (2005) 322329.

[26] J.M. Fernández Oro, K.M. Argüelles Díaz, C. Santolaria Morros, E. Blanco Marigorta, Unsteady flow and wake transport in a low-speed axial fan with inlet guide vanes, ASME Journal of Fluids Engineering 129 (2007) 1015-1029.

[27] S.B. Pope, Turbulent Flows, Cambridge University Press, 2000.

[28] E.M. Greitzer, C.S. Tan, D.C. Wisler, J.J. Adamczyk, A.J. Strasizar, Unsteady flows in turbomachines: where's the beef?. Proceedings of the 1994 ASME Winter Annual Meeting, Chicago, Nov. 1994.

[29] R. Ballesteros, E. Blanco, C. Santolaria, Blade pitch influence at the exit flow field of an axial flow fan, ASME 95GT-191.

[30] S. Velarde-Suarez, R. Ballesteros-Tajadura, C. Santolaria-Morros, E. Blanco-Marigorta, Total unsteadiness downstream of an axial flow fan with variable pitch blades, ASME Journal of Fluids Engineering 124 (2002) 280-283.

[31] J. Lewalle, D.E. Ashpis, Estimation of time scales in unsteady flows in a turbomachinery rig. Final Report NASATM-2004-209452, NASA Lewis Research Center, Cleveland, Ohio, USA, 2004.

[32] E. Blanco, R. Ballesteros, C. Santolaria, Angular range and uncertainty analysis of non-orthogonal crossed hot wire probes, ASME Journal of Fluids Engineering 123 (1998) 9094.

[33] L. Porreca, M. Hollestein, A.I. Kalfas, R.S. Abhari, Turbulence measurements ans analysis in a multistage axial turbine, Proceedings of the 17th International Symposium on Air Breathing Engines, ISABE, Munich, 4-9 September, 2005. 


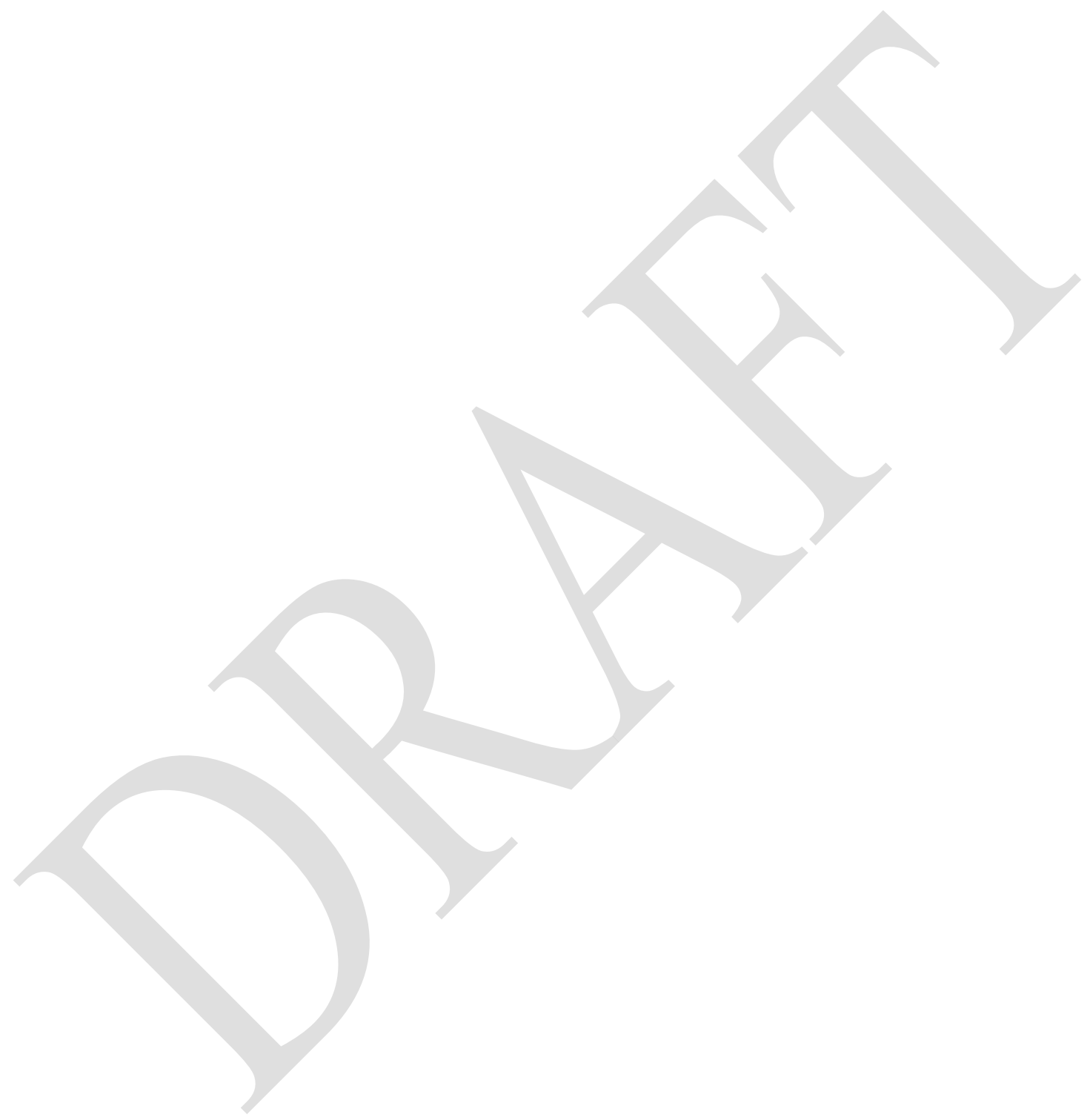


This document is a pre-print version of the scientific paper published by Elsevier. It has been released by the authors to fulfill all the publisher requirements established for Article Sharing: https://www.elsevier.com/about/policies/sharing

\section{(9) $\Theta \Theta \Theta$}

(C) 2019. This manuscript version is made available under the Creative Commons Attribution-NonCommercial-NoDerivatives 4.0 International License (CC-BY-NC-ND 4.0 license) http://creativecommons.org/licenses/by-nc-nd/4.0/ 